\title{
Wirtschaften im Land der unbegrenzten Unmöglichkeiten?
}

\author{
Axel Schubert ${ }^{*}$
}

„Rußland ist das Land der unbegrenzten Unmöglichkeiten“, spottete mein Reisebegleiter, während einer höchst ungewöhnlichen Befragung durch Mitglieder der OMON Miliz auf dem Kasaner Bahnhof in Moskau. Die Ordnungshüter hatten die Zugänge der Bahnsteige abgesperrt und kontrollierten das Gepäck aller Reisenden. Gesucht wurde nicht nach Waffen oder Drogen, sondern nach Handelswaren, deren Wert 200 US\$ überstieg. Für diese, so ein kurzfristiger Beschluß der Russischen Eisenbahnverwaltung, sei ein gesondertes Beförderungsentgeld zu entrichten. Bereits eine Woche später gehörten die Kontrollen der Vergangenheit an. Derartige Begebenheiten, die hierzulande als Reiseanekdoten die Zuhörer immer wieder belustigen und die in Westeuropa gängigen Klischees bestätigen, sind symptomatisch für alle Bereiche der russischen Gesellschaft. Besonders prekär sind ihre Auswirkungen im Bereich der Wirtschaft. Nichtdestotrotz bleibt Rußland das „Land der unbegrenzten Unmöglichkeiten“, da sich der Markt im Umbruch befindet. Bei allen Risiken und Schwierigkeiten erscheinen die Entwicklungschancen für ausländische Unternehmen attraktiv.

\section{Gründung von Unternehmen}

Der juristische Akt der Gründung und Registrierung ausländischer oder gemischter Unternehmen in Rußland ist unkompliziert. Selbst Aktiengesellschaften lassen sich in Monatsfrist konstituieren.

Ungleich schwieriger hingegen die ersten Schritte zur Aufnahme der geregelten Geschäftstätigkeit: Bei der Eröffnung von Firmenkonten muß zum Beispiel neben einer Genehmigung der Steuerbehörde ein Nachweis über die Registratur des Unternehmens bei einem sogenannten Pensionsfond, einer mit den Rentenkassen in Deutschland vergleichbaren Institution, erbracht werden. Der Zeitaufwand für dieses Papier kann unter Umständen eine Woche in Anspruch nehmen.

Axel Schubert, geb. 1969, Student der Politikwissenschaft an der TU Chemnitz, führte von 1993 bis 1995 die Geschäfte eines Deutsch-Russischen Handelsunternehmens in Nishnij Novgorod. 


\section{Bürokratie}

Große Schwierigkeiten bereitet nicht nur Ausländern der Umgang mit den verschiedenen Vertretern der russischen Bürokratie. Exemplarisch ist die „Zusammenarbeit“ mit den Zoll- und Steuerbehörden.

Unabhängig vom Umfang von Warenlieferungen sollten Unternehmen, die keine besonderen oder privaten Beziehungen zu Beamten des russischen Zolls unterhalten, mit Abwicklungsfristen von mindestens einer Woche rechnen, da nicht nur die üblichen Zolldokumente und Zertifikate erbracht werden müssen, sondern zusätzlich Bescheinigungen der staatliche Kommission zur Kontrolle von Devisengeschäften, der Steuerbehörde und gegebenenfalls der russischen Industrie- und Handelskammer. Der Zweck der Bescheinigungen bleibt dabei oft im Dunkeln, da nach der anfänglichen und bisweilen zeitraubenden Weitergabe der Vorgänge innerhalb der Verwaltungsebenen die Papiere in der Regel problemlos ausgestellt werden.

Eine Frage der innerbetrieblichen Organisation ist zudem damit verbunden: Einerseits ist der Zeitaufwand für Behördenkontakte prinzipiell so groß, daß es unrentabel ist, Mitarbeiter des europäischen Stammhauses einzusetzen, zumal diese in der Regel Geduld und Demut, mit denen man in russischen Behörden erfolgreich ist, nicht aufbringen. Andererseits wertet der Besuch eines „hochrangigen Ausländers“ den Status des Mitarbeiters oder Vorstehers der jeweiligen Behörde auf und erleichtert künftig die Zusammenarbeit. „Schlimmstenfalls“ muß der „Stammhausdelegierte“ mit einem improvisierten Bankett nach Feierabend rechnen. Er kann dann aber künftig selbst größere Probleme telefonisch regeln.

\section{Zoll- und Steuergesetzgebung}

Ein Problem entsteht durch die Unbeständigkeit der juristischen Situation im genannten Bereich. Zollbestimmungen ändern sich willkürlich und treten nicht selten sofort in Kraft. Nicht nur die langfristige Planung wird dadurch unmöglich auch bereits laufende Geschäfte werden so akut gestört.- Als Alternative, zu einem Geschäft über zwei Eisenbahncontainer Bier, die den Grenzbahnhof erst erreichten als eine neue Akzise in Kraft getreten war, riet mir ein ehemaliger Mitarbeiter des Außenhandelsministeriums der Sowjetunion „Fisch zu importieren“. Gemeint war damit, die Lieferung in die Freihandelszone Kaliningrad zu bringen und von dort in einem Fischtrawler nach St. Petersburg zu schmuggeln. Selbstverständlich lassen sich so seriöse, langfristige Geschäftskontakte nicht realisieren.- Andere Möglichkeiten, den restriktiven Bestimmungen in Rußland zu entgehen, gibt es nicht.

Ähnlich verhält es sich im Bereich der Steuergesetzgebung: Zu Beginn des Jahres 1994 traten in Rußland verschärfte Gesetze im fiskalischen Bereich in Kraft, die einerseits dem privatwirtschaftlichen Sektor erhebliche Schwierigkeiten bereiteten und andererseits Wirtschaftsbeziehungen zwischen 
den Unternehmen des Privatsektors und ihren ausländischen Partnern störten. So können Kosten erst nach dem Verkauf der Ware geltend gemacht werden, für deutsche Begriffe ein Verstoß gegen das Gebot der kaufmännischen Vorsicht. Eine Auswirkung ist: Betriebe werden gezwungen, in der für alle Unternehmen obligatorischen Vierteljahresbilanz mehr Vermögen als tatsächlich vorhanden auszuweisen und zu versteuern. Die dem Staat so zugute kommende Fiskalrente schmälert die Liquidität der Unternehmen und hat nicht selten zur Folge, daß die Firmen falsche Bilanzen Steuerunterlagen einreichen, was letztlich eine teilweise Abwanderung in den Bereich illegaler Geschäfte zur Folge hat. Dem Beobachter drängt sich der Verdacht auf, daß die Kriminalisierung Teil des Konkurrenzkampfes zwischen neuen Wirtschaftstrukturen und den Nachfolgern der sowjetrussischen Trusts ist, da letztere erhebliche Steuerprivilegien genießen.

\section{Unternehmenskultur}

Bemerkenswert ist die emotionale Bindung russischer Mitarbeiter an ihr Unternehmen. Oft wird sein Erfolg mit dem eigenen Schicksal gleichgesetzt. Die Ursachen dafür liegen einerseits in der Tatsache, daß es, aufgrund der wirtschaftlichen Situation Rußlands, für Beschäftigte in ausländischen Firmen keine Alternative oder Steigerung mehr gibt. Andererseits ist die Neigung zu emotionellen Reaktion sozio- kultureller Natur. So spielen Titel und Direktorenposten für alle sinnigen und unsinnigen Bereiche oft eine größere Rolle, als das Einkommen, vorausgesetzt, dieses erreicht die zum Überleben notwendige Grenze.

Sicher fehlt besonders älteren Mitarbeitern die Bereitschaft und möglicherweise die Fähigkeit, selbständig und eigenverantwortlich Entscheidungen zu treffen. Die vergewissernde Nachfrage beim Chef ist jedoch nicht nur Ausdruck der eigenen Unsicherheit, sondern hat auch die Funktion, auf eigene Gedanken aufmerksam zu machen und ein Feed Back zu erhalten, welches über das z.B. in Arbeitsbesprechungen übliche Maß hinausgeht, den persönlichen Kontakt zu forcieren.

Ausländischen Mitarbeitern wird besonders bei der Lösung von Sachfragen die größere Kompetenz zugeschrieben. Störend im Betriebsklima ist die Tatsache, daß Ausländer nicht als Bestandteil der Umwelt angesehen werden, sondern Gäste sind, die es unter Umständen vor Schwierigkeiten zu bewahren gilt. Ein kollegiales, gleichberechtigtes Verhältnis, für europäische Unternehmen die Grundlage der Führungskonzeption, ist daher in Firmen mit gemischten Belegschaften nur schwer zu erreichen.

Da diese Probleme historische Wurzeln haben und nicht nur eine Folge des unter dem kommunistischen Regime praktizierten „Demokratischen Zentralismus“ sind, empfiehlt es sich bei der Ausarbeitung von Führungskonzepten, nicht auf 
Änderungen der Verhaltensweisen zu drängen, sondern sie als gegebene Größe zu akzeptieren.

\section{Fazit}

Die erfolgreiche Teilnahme an den „unbegrenzten Unmöglichkeiten“ der russischen Gesellschaft, das erfolgreiches wirtschaftliche Engagement in Rußland, setzen die Trennung von „europäisch- abendländischem Denken und Begreifen“ voraus. Es geht in Rußland nicht darum, einen Umschwung im Lande in Richtung „westliche Zivilisation“ zu erwarten. Der wäre historisch gesehen ein Novum. Die Versuche, Russland zu europäisieren, selbst wenn sie von Russen unternommen wurden, waren immer Gegenstand härtester Auseinandersetzungen oder wurden bereits nach kurzer Zeit revidiert. Dostojewski, selbst ein Gegner der „Verweltlichung“, machte die russische Seele sichtbar, ohne deren Verständnis Russland nicht zu erfassen ist. Europäische Rußlandkenner irrten sich immer dann, wenn eingeschätzt wurde, daß Rußland nun reif für eine enge Zusammenarbeit mit dem Westen wäre. Das letzte Mal traten diese Prognosen nicht ein, als nach der Verkündung der „Neuen Ökonomischen Politik“ zu Beginn der zwanziger Jahre, ein internationales Investorenkonsortium gegründet wurde, das unter diesen neuen Umständen trotz Bürgerkrieg und militärischer Interventionen die Möglichkeit der Zusammenarbeit mit der Sowjetunion sah. Was daraus wurde, ist mittlerweile bekannt... 